\title{
A density matrix renormalization group study of low-lying excitations of polythiophene within a Pariser-Parr-Pople model
}

\author{
MOSUMI DAS and S RAMASESHA* \\ Solid State and Structural Chemistry Unit, Indian Institute of Science, Bangalore 560012 \\ e-mail: ramasesh@sscu.iisc.ernet.in
}

\begin{abstract}
Symmetrized density-matrix-renormalization-group calculations have been carried out, within Pariser-Parr-Pople Hamiltonian, to explore the nature of the ground and low-lying excited states of long polythiophene oligomers. We have exploited $C_{2}$ symmetry and spin parity of the system to obtain excited states of experimental interest, and studied the lowest dipole allowed excited state and lowest dipole forbidden two photon state, for different oligomer sizes. In the long system limit, the dipole allowed excited state always lies below the lowest dipole forbidden two-photon state which implies, by Kasha rule, that polythiophene fluoresces strongly. The lowest triplet state lies below two-photon state as usual in conjugated polymers. We have doped the system with a hole and an electron and obtained the charge excitation gap and the binding energy of the $1^{1} B_{u}^{-}$exciton. We have calculated the charge density of the ground, one-photon and two-photon states for the longer system size of 10 thiophene rings to characterize these states. We have studied bond order in these states to get an idea about the equilibrium excited state geometry of the system. We have also studied the charge density distribution of the singly and doubly doped polarons for longer system size, and observe that polythiophenes do not support bipolarons.
\end{abstract}

Keywords. DMRG technique; PPP Hamiltonian; low-lying excited states; fluorescence; Kasha rule.

\section{Introduction}

One of the most successful of conjugated systems for applications in optoelectronic devices are thiophenes and thiophene oligomers $(n \mathrm{~T}, n$ is the number of thiophene rings). ${ }^{1}$ Chemical stability and the solubility of the alkyl-substituted polythiophenes (PT) in common solvents make them suitable for diverse technological applications ${ }^{2-5}$ such as batteries, optoand thermochromic devices, light-emitting diodes, ${ }^{6,7}$ plastic lasers, ${ }^{8,9}$ photovoltaic cells ${ }^{10,11}$ and more recently, field-effect transistors; $\alpha$-sexithienyl $(\alpha-6 \mathrm{~T})$ has been successfully used as an active semiconducting material in thin-film transistors. ${ }^{12-14}$

Polythiophene serves as a model system for understanding the electronic and optical properties of onedimensional systems with nondegenerate ground states. The conjugated PT backbone resembles cis$(\mathrm{CH})_{x}$ suggesting that the presence of sulphur atom is a small perturbation on the cis-polyenes. ${ }^{2,3}$ Hence it is interesting to compare the results from quantum chemical and spectroscopic investigations of thio-

Dedicated to Prof J Gopalakrishnan on his 62nd birthday *For correspondence phene oligomers with that of polyenes. It has been observed that the two-photon absorption spectra and the fluorescence spectra of polythiophene are quite different from those of polyenes. ${ }^{15-18}$ The lowest singlet excitation $S_{1}$ of polyenes is the two-photonallowed $2 A_{g}$ state, while $S_{1}$ for PT is the dipoleallowed $1^{1} B_{u}$ state. Kohler et $a l^{19-22}$ carried out high resolution spectral investigations on thiophene oligomers from $2 \mathrm{~T}$ to $4 \mathrm{~T}$ at low temperature. They concluded from their results that ${ }^{1} B_{u}$ state is the lowest excited state while $2^{1} A_{g}$ state has been located above ${ }^{1} B_{u}$. The $2^{1} A_{g}$ state also has been located above the $1^{1} B_{u}$ in a crystalline film of $\alpha-6 \mathrm{~T} .{ }^{15}$ The thiophene molecule was first studied theoretically in 1935 using the Hückel model. ${ }^{23,24}$ Theoretical studies of the lowest excited states of thiophene oligomers have been carried out in recent times. ${ }^{25-27}$ Calculations by Beljonne et $a l^{25}$ predicted that $2^{1} A_{g}$ state is below the ${ }^{1} B_{u}$ state till $4 \mathrm{~T}$ and crossover occurs between $4 \mathrm{~T}$ and 5T. Still other calculations on $2 \mathrm{~T}$ and some higher $n \mathrm{~T}$ give varying results regarding energy ordering of the states for $2 \mathrm{~T}$ and place the $2^{1} A_{g}$ state lowest for $3 \mathrm{~T}$ and $4 \mathrm{~T}^{26,27}$ In view of such diverse contradictory results relating to energy level ordering, Colditz et al carried out theoretical CNDO/S calcu- 
lations including single and double excited configurations on thiophene oligomers, $n \mathrm{~T}$, with 2 to 6 monomer units. ${ }^{28}$ Their theoretical studies and spectroscopic investigation of the ground and excited electronic states of thiophene oligomers showed that the $2^{1} A_{g}$ lies above the $1^{1} B_{u}$ state. Becker et al came up with experimental evidence of the fact that the state $1^{1} B_{u}$ is the lowest excited state in all $n$-oligothiophenes from $2 \mathrm{~T}$ to $7 \mathrm{~T}^{29}$

Semi-empirical calculations have not been able to provide a clear picture of the electronic spectrum. ${ }^{30-34}$ While theoretical studies on thiophene molecule reported are based on ab initio quantum chemical approach, ${ }^{35-37}$ due to its large size, similar studies on PT is difficult using these methods. Restricted configuration interaction (CI) technique is also not useful for solving these interacting systems as we need to extrapolate the results to the polymer limit and restricted CI calculation are not size consistent. PT has conjugated $\mathrm{sp}^{2}$ backbone and hence can be numerically accurately described by the Pariser-Parr-Pople (PPP) model, which includes explicit long-range electron-electron interactions. ${ }^{38,39}$ However, the interaction parameters for sulphur need to be optimised. In our studies reported here, we have employed the density matrix renormalization group (DMRG) method for large oligomers of polythiophene systems within the PPP model. ${ }^{40}$ The DMRG method has proved to be the best choice for one-dimensional as well as quasi-one-dimensional systems. ${ }^{41}$ The excited states of the polythiophene systems have been calculated through symmetrized DMRG technique developed by Pati et $a l .{ }^{42}$ We have also calculated the excitation energy gaps of low-lying excited states. Besides, we have computed properties like bond-order and chargedensities to characterize these states. In what follows we briefly discuss the PPP model and its parameters in the next section. In $\S 3$ we discuss the method of implementing the DMRG scheme for polythiophene. In $\S \S 4$ and 5 we discuss the results for neutral and doped polythiophenes and conclude the paper in $\S 6$.

\section{The PPP model Hamiltonian and computational scheme}

The PPP Hamiltonian in second quantized notation can be written as,

$$
H_{P P P}=\sum_{i j, \sigma} t_{i j}\left(\hat{a}_{i \sigma}^{\dagger} \hat{a}_{j \sigma}+H . c .\right)+\sum_{i} \hat{n}_{i} \varepsilon_{i}
$$

$$
+\frac{U}{2} \sum_{i} \hat{n}_{i}\left(\hat{n}_{i}-1\right)+\sum_{i, j} V_{i, j}\left(\hat{n}_{i}-z_{i}\right)\left(\hat{n}_{j}-z_{j}\right)
$$

where the symbols have their usual meaning and the intersite interaction potential $V_{i j}$ is parametrized using Ohno parametrization. ${ }^{43}$ The value of $z_{C}$ for carbon atoms is one while for sulphur $z_{S}=2$, since the lone pair on sulphur is involved in conjugation. We have carried out all our calculations using experimentally determined ground state geometry with $C_{2 v}$ symmetry. ${ }^{44}$ Figure 1 shows two thiophene rings in the $X-Y$ plane and the $C_{2}$ axis is perpendicular to this plane. The conjugated carbon backbone in polythiophene backbone resembles cis-polyacetylene $\left((\mathrm{CH})_{x}\right),{ }^{45}$ sulphur atoms have been treated as non-conjugated heteroatoms producing a charge-density-wave (CDW) ground state. Soos et $a l^{27}$ modelled the one and two photon excitations of polythiophene in terms of interacting $\pi$-electrons and a charge-density-wave (CDW) ground state due to sulphur atoms. ${ }^{27}$ They have incorporated the effect of sulphur atoms by introducing effective orbital energy or site energy at carbon sites. It is seen that if we assume the linear polyene structure discussed above with an effective orbital energy without explicitly using the sulphur atom parameters for $U$ and $t$ and carry out the calculation within the PPP model, experimental excitation energies cannot be reproduced. So it is essential to include sulphur atoms explicitly during thiophene oligomer calculation.

The parameters required for calculation are transfer integral for the $\mathrm{C}-\mathrm{S}$ bond $t_{\mathrm{C}-\mathrm{S}}$, the on-site correlation energy, $U_{\mathrm{S}}$, for sulphur, the site or orbital energy $\varepsilon_{\mathrm{S}}$ for sulphur relative to carbon. The study of conjugated organic polymers without any heteroatoms has shown that the $\pi$-orbital of the $s p^{2}$ carbon atom has transferable parameters with transfer integral $2.4 \mathrm{eV}$ for a C-C separation of $1.397 \AA$ and onsite repulsion

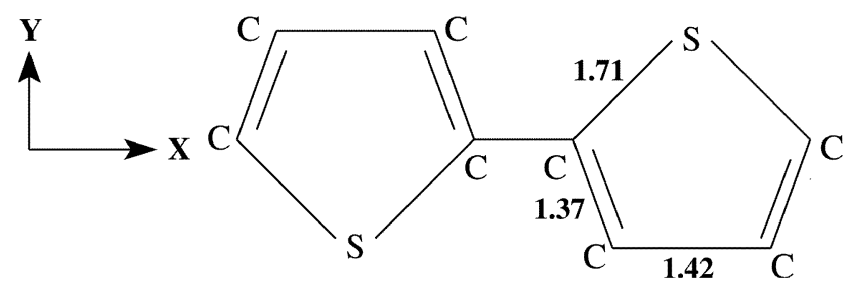

Figure 1. Schematic representation of two thiophene rings (2T) in $X-Y$ plane. $\mathrm{C}-\mathrm{C}$ and $\mathrm{C}-\mathrm{S}$ bond lengths are in $\AA$. 

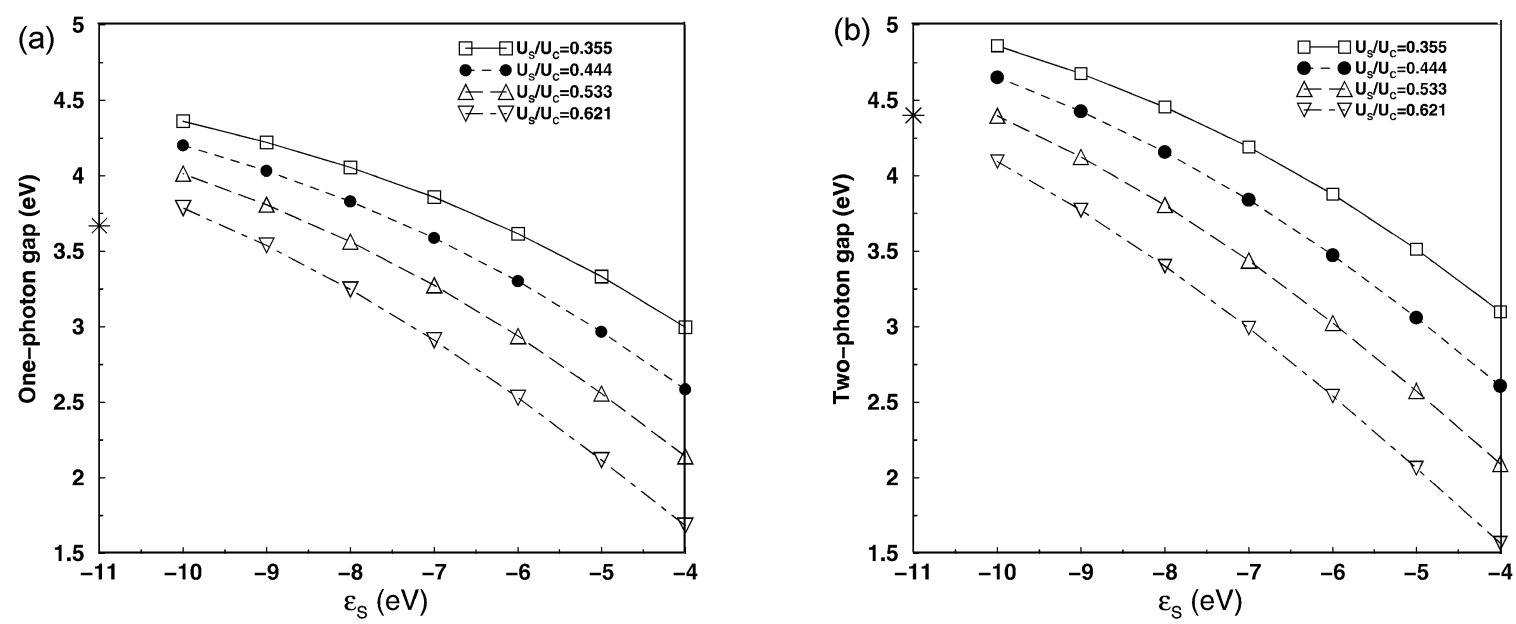

Figure 2. Figure shows the variation of one-photon (a) and two-photon energy gap (b) as a function of different values of $\varepsilon_{\mathrm{S}}$ at four sets of $U_{\mathrm{S}}=U_{\mathrm{C}}$ for two-rings of thiophene (2T). The experimental values of one- and two-photon gaps which are independent of the $X$-axis are shown by a star symbol on the $Y$-axis. We can see from the two plots that the calculated and corresponding experimental values are comparable at $U_{\mathrm{S}}=U_{\mathrm{C}}=0.444$ and $\varepsilon_{\mathrm{S}}=-8 \cdot 0$. We have chosen $\varepsilon_{\mathrm{S}}=-7 \cdot 8$ to make the one-photon gap closer to the experimental value.

$11 \cdot 26 \mathrm{eV}$. For the $\mathrm{C}-\mathrm{C}$ bonds at other distances, transfer integral $t_{\mathrm{C}-\mathrm{C}}$ is approximated by, ${ }^{46}$

$$
t_{\mathrm{C}-\mathrm{C}}=-2 \cdot 4+3 \cdot 20\left(r_{\mathrm{C}-\mathrm{C}}-1 \cdot 397\right)
$$

where the transfer integral is in $\mathrm{eV}$ and the $\mathrm{C}-\mathrm{C}$ distance is in $\AA$. To determine the PPP parameters for sulphur, we have carried out exact valence bond calculation for the two thiophene rings (2T) shown in figure 1, taking the experimentally determined ground state geometry of thiophene molecule. ${ }^{44}$ We have compared the excitation energies with experimental values reported by Birnbaum et al. ${ }^{19}$

We have varied the parameters, $U_{\mathrm{S}}, \varepsilon_{\mathrm{S}}$ and $t_{\mathrm{C}-\mathrm{S}}$, and compared the calculated excitation energies with the corresponding experimental values. The sulphur atom lies in the 3rd row of the periodic table and hence we expect the $3 p$ orbitals of sulphur to be more diffuse than the carbon $2 p$ orbitals. Therefore the Hubbard $U$ on sulphur is expected to be smaller than $U_{\mathrm{C}}$. Since orbital energies decrease with increasing atomic numbers, we also expect the sulphur $3 p$ orbital to be having a lower orbital energy than the $\mathrm{C}_{2 p}$ orbital. The more diffuse $3 p$ orbital also implies a larger $t_{\mathrm{C}-\mathrm{S}}$ than $t_{\mathrm{C}-\mathrm{C}}$ for the same separation of the atom pairs. We have searched for the parameters of $U_{\mathrm{S}}$ and $\varepsilon_{\mathrm{S}}$ based on these physical arguments. We have shown the variation of the one-photon $\left(1^{1} B\right)$ and two-photon $\left(2^{1} A\right)$ gaps for two thiophene rings (2T) obtained using exact diagrammatic valence bond ${ }^{47}$ method as a function of the parameters in fig- ure 2. By keeping the $t_{\mathrm{C}-\mathrm{S}}$ value to be fixed at $3.0 \mathrm{eV}$, we wish to choose $U_{\mathrm{S}}$ and $\varepsilon_{\mathrm{S}}$ values that reproduce the one-photon gap the best. We find that $U_{\mathrm{S}}=5 \mathrm{eV}$ and $\varepsilon_{\mathrm{S}}=-7.8 \mathrm{eV}$ well reproduce the onephoton gap while the two-photon gap is in error by only $0.4 \mathrm{eV}$. In view of this, we employ these sulphur parameters in all our calculations.

\section{DMRG method for polythiophene}

While implementing DMRG technique to polythiophene within PPP model we see that the interaction part although diagonal in real space representation is truly long-ranged and the topology of these interactions correspond to that of a higher dimensional system. Notwithstanding this higher effective dimensionality, because the interactions are diagonal, the DMRG technique has proved to be highly accurate. It appears that the DMRG technique loses its accuracy only when the off-diagonal interactions are of higher dimensional topologies. The polythiophene oligomers, in the DMRG procedure, are built up two sites at a time. The sites are added in such a manner that only short-ranged transfers (transfer operator between recently added sites) are introduced at every iteration. This is possible since we have chosen to buildup the system from the interior. The polythiophene oligomers are encountered for total number of sites corresponding to integer multiples of ten. Schematically we have shown the way in which the polythiophene oligomers are constructed in DMRG scheme 
in figure 3. It is required to use symmetries like spatial symmetry and spin parity symmetry to study optical excitations or spin excitations in such conjugated polymer systems. In the study of polythiophene we employ (i) $C_{2}$ symmetry and (ii) spin parity. The polythiophene chain has $C_{2}$ symmetry or reflection symmetry about an axis perpendicular to the plane containing the molecule. We use here spin parity that partitions the Hilbert space of the system into even and odd parity total spin sectors. Electron-hole symmetry is broken both because of the presence of a heteroatom and also because of the thipohene moiety present in the oligomers. The implementation of $C_{2}$ symmetry and spin parity symmetry in the DMRG procedure gives the ground state which is in A subspace and has even spin parity ${ }^{e} A$. The optically allowed state which is dipole allowed with the covalent ground state lie in the even parity ${ }^{e} B$ subspace known as one-photon state. The two-photon state $2^{e} A$ also has even spin parity. The triplet states lie in
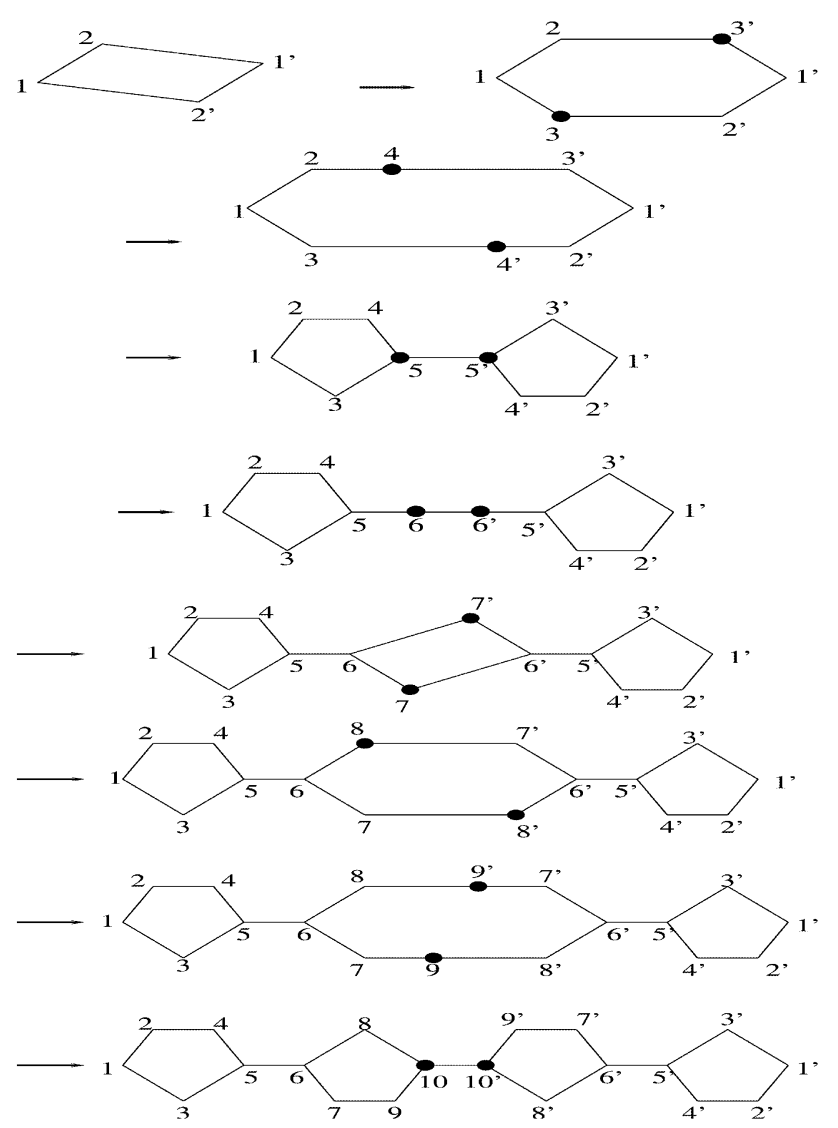

Figure 3. Scheme for building up polythiophene oligomers, adding two sites at a time starting from a four-site system in the DMRG procedure. The primed sites correspond to the right block and the unprimed sites to the left block. the odd parity (o) subspace. We start the DMRG process with the initial system size of four sites and build up the oligomers of thiophene.

To improve the accuracy of the energy values, we have employed the average density matrix approach in our calculation. We have calculated the density matrix for the low-lying states in the $A$ and $B$ subspaces. The average of these two density matrices is used for obtaining the highest $m$ eigenvalues and eigenvectors used in the renormalization. To compute properties like charge densities and bond orders, we have followed the numbering scheme shown in figures 4 and 5 respectively. The largest system size we have studied is one with ten thiophene rings.

\section{Results and discussion}

Our aim is to study the nature of the ground and a few low-lying excited states for a series of oligomers and to obtain the excitation gaps in the thermodynamic limit. We have employed the symmetrized DMRG technique to calculate the ground state and excited states for all system sizes, retaining 128 density matrix eigenvectors and employing the infinite DMRG algorithm. In order to establish the accuracy of our DMRG procedure, we compare the DMRG results for smaller systems (up to two thiophene monomers) with the results obtained from exact diagonalization calculations within the PPP model Hamiltonian. ${ }^{47}$ Table 1 shows the comparison of DMRG energies with exact energies for ground state, the lowest one-photon dipole allowed and twophoton states of two thiophene rings for the PPP model. We note that all the three energies are well reproduced by the DMRG method. We have also calculated bond orders, charge densities and find that they all compare well with the exact numbers.

To evaluate the DMRG method for larger system sizes, we have obtained excitation energy gaps from Hückel model calculation for system sizes of up to ten thiophene rings. In figure 6 , we show the variation of one-photon gap for the exact and DMRG calculations as a function of system size. From the figure, we observe that even for the noninteracting case where the DMRG accuracy is generally poor, the DMRG optical gaps compare favourably with exact results. We have calculated various excitation energy gaps, expectation values and transition dipole moments between dipole-allowed states. The size of the system considered is sufficient to saturate most important low-lying excitation gaps, so that all 


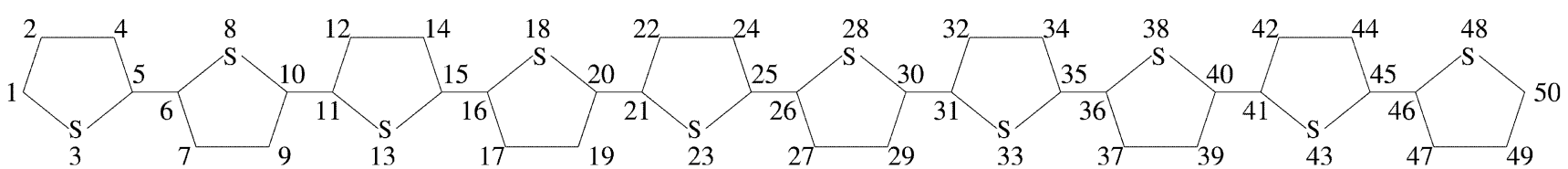

Figure 4. Numbering of atoms (sites) for polythiophene.

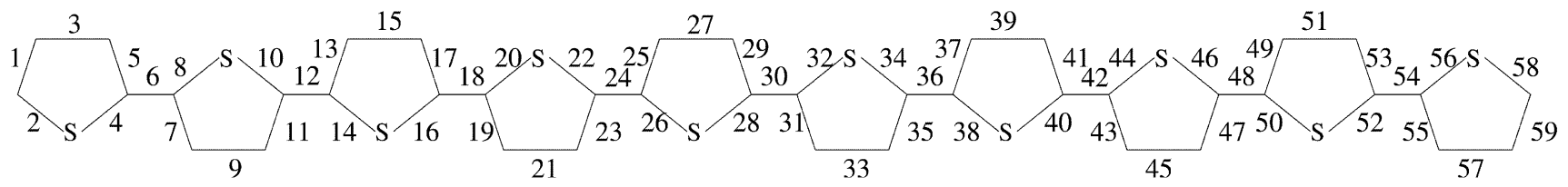

Figure 5. Numbering of bonds for polythiophene.

Table 1. Comparison of DMRG and exact energies for ground state, one-photon dipole allowed state and twophoton state for two rings of thiophene within PPP model Hamiltonian

\begin{tabular}{lcc}
\hline $\begin{array}{l}\text { State } \\
\text { symbol }\end{array}$ & $\begin{array}{c}\text { Exact energy } \\
(\mathrm{eV})\end{array}$ & $\begin{array}{c}\text { DMRG energy } \\
(\mathrm{eV})\end{array}$ \\
\hline $1^{1} A$ & -43.762 & -43.761 \\
$1^{1} B$ & -39.976 & -39.973 \\
$2^{1} A$ & -39.664 & -39.659 \\
\hline
\end{tabular}

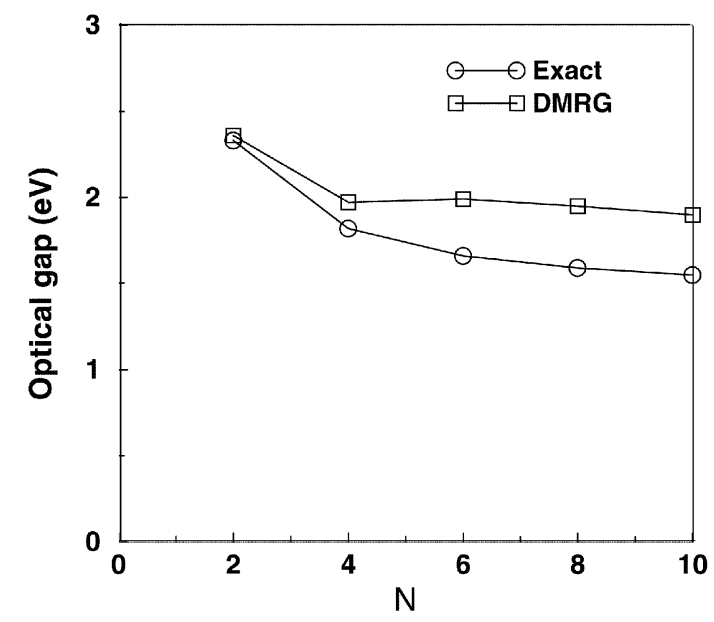

Figure 6. Comparison of the optical gap from exact Hückel calculation with those obtained from DMRG calculations for polythiophene with 10 rings.

the above properties can be evaluated in the polymer limit, by extrapolation. We have also studied quantities such as exciton and bipolaron binding energy by obtaining ground state energy of the singly and doubly doped polythiophene systems.

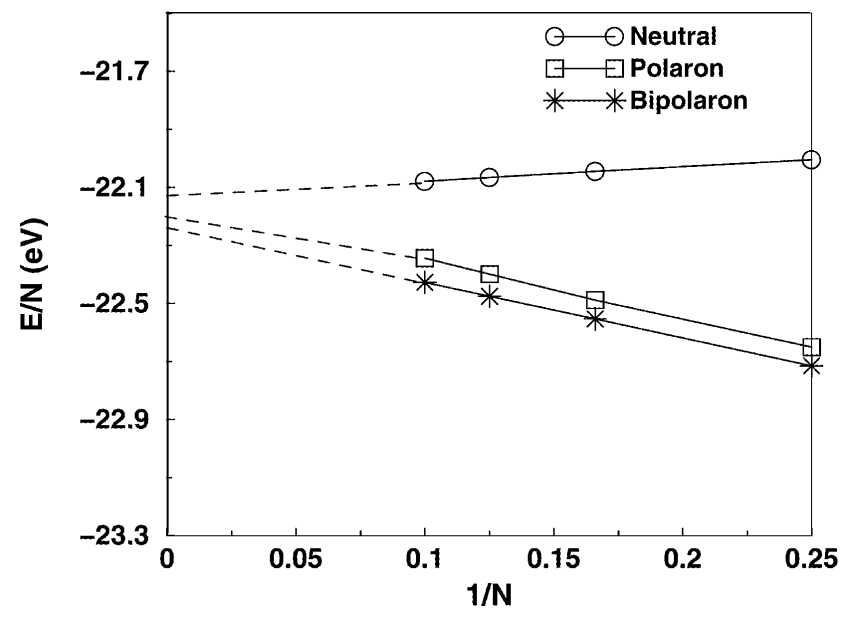

Figure 7. Energy per thiophene ring as a function of inverse system size for neutral, singly and doubly holedoped polythiophene.

We find that irrespective of the system size the ground state lies in the ${ }^{e} A$ subspace. We have plotted in figure 7 the ground state energy per thiophene monomer as a function of inverse of number of monomers, for the neutral, the one and two electron doped systems. The linear dependence on $1 / N$ allows extrapolation of the energy per monomer to the thermodynamic limit. The energy per monomer converges well showing that it as an intensive variable. In the thermodynamic limit, the differences in the energy between neutral and doped systems are finite, hence we should expect to obtain the same value for energy per monomer in all cases. We indeed get nearly the same value; the discrepancy is an estimate of the error and we find the extrapolation error to be less than $1 \%$. We have calculated the excitation en- 
ergy gaps from the ground state to various low lying excited states of interest, namely the lowest one photon state $\left(1^{1} B\right)$, the lowest two-photon state $\left(2^{1} A\right)$, and the lowest triplet energy state $\left(1^{3} B_{u}\right)$ for oligomers with up to 10 thiophene rings. Figure 8 shows the variation of optical gap, two-photon gap, spin gap as a function of inverse system size. All these gaps show very weak dependence on the system size and saturate at about four thiophene rings. The plot shows that the $1^{1} B$ state is well below $2^{1} A$ for all oligomers. These thiophene oligomers should strongly

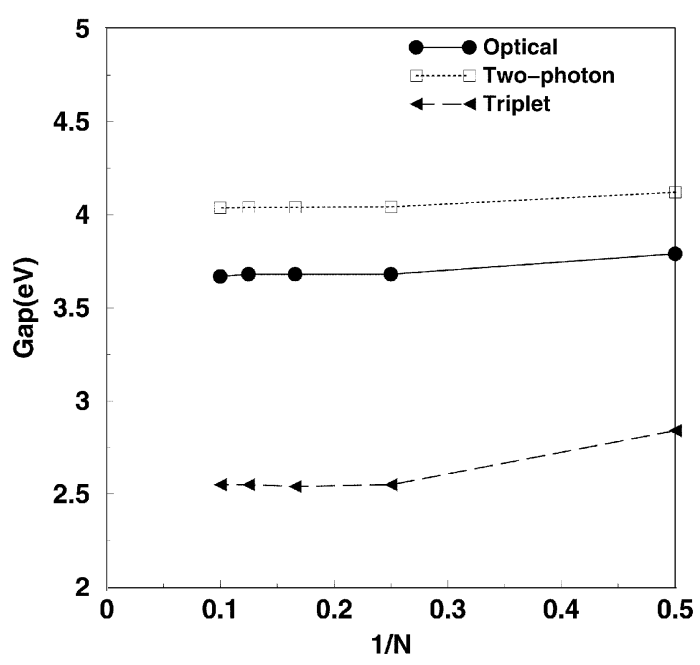

Figure 8. Energy gaps in the PPP model to the lowest triplet (filled triangles), dipole-allowed (circles) and twophoton (squares) states for polythiophene. The plot shows the linear extrapolation of these gaps to infinite system size.

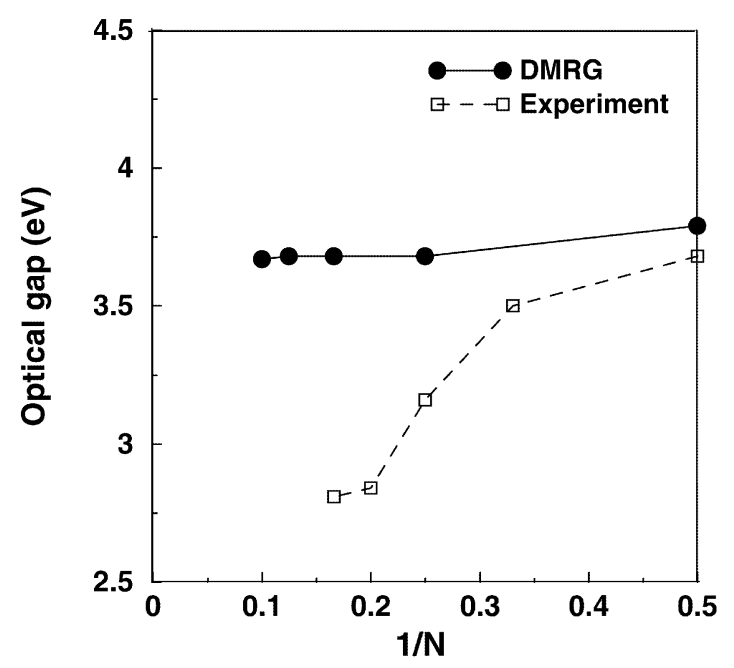

Figure 9. The plot shows the optical gaps for polythiophene from theory and optical-absorption measurement. ${ }^{29}$ fluoresce according to Kasha's rule ${ }^{48}$ which is indeed found to be the case experimentally by Becker et $a l .{ }^{29}$ The energy ordering of $2^{1} A$ and $1^{1} B$ states is reversed compared to that of polyene, making these thiophene oligomers strong fluorescent molecules. We can extrapolate these gaps in figure 8 to obtain their values in the polymer limit. The extrapolated values of optical, spin and two-photon gaps are 3.67, 2.55 and $4.04 \mathrm{eV}$ respectively. Thienpont et al, ${ }^{49}$ from optical-absorption measurements on well-characterized alkyl-substituted oligothiophenes, found that the optical gap does not vary beyond a system size of 6 thiophene rings. They found that the saturation value of optical gap is $2.78 \mathrm{eV}$. The DMRG value for optical gap at the thermodynamic limit is $3.67 \mathrm{eV}$ which is higher, but discrepancy could be attributed to the fact that the experiments were carried out in solution. The ordering of energy levels obtained from theory is the same as that found experimentally. The two-photon gap was studied experimentally by Periasamy et al. ${ }^{15}$ Their studies place the two-photon state at about $0 \cdot 1 \mathrm{eV}$ above the optical gap. We see from figure 8 that the extrapolated spin gap which is $2.55 \mathrm{eV}$ is $\approx 1.0 \mathrm{eV}$ less than the optical gap. Monkman et $a l^{50}$ find that for poly(3-octyl thiophene), the energy difference between the lowest triplet state and the lowest singlet excited state, is $1.18 \mathrm{eV}$, which compares well with the DMRG value of $1.12 \mathrm{eV}$, in the thermodynamic limit. There have been earlier quantum chemical calculations by Colditz et al. ${ }^{28}$ These calculations at the SCI level place the two-photon state, about 0.7$0.8 \mathrm{eV}$ above the optical gap, while the SDCI calculations indicate a crossover of the $1 B-2 A$ levels with system size. We have computed the transition dipole moment for 7 lowest singlet states in the $B$ space from the ground state for thiophene oligomers with up to 10 monomers. The square of the transition dipole mo-ment, which is proportional to the intensity, has been plotted as a function of the oligomer size, in figure 10 . We note that up to about $7 \mathrm{eV}$, we find the $1^{1} B$ state to be the dominant dipole coupled state. The magnitude of the transition dipole does not change with system size beyond six monomers indicating that the $1^{1} B$ excitation is fairly localized.

To further characterize the eigenstates, we have obtained the charge density and bond order for ground state as well as excited states. In figure 11 we have plotted the difference in charge density of one-photon state, two-photon state and lowest triplet state with respect to the ground state. We see that rearrange- 


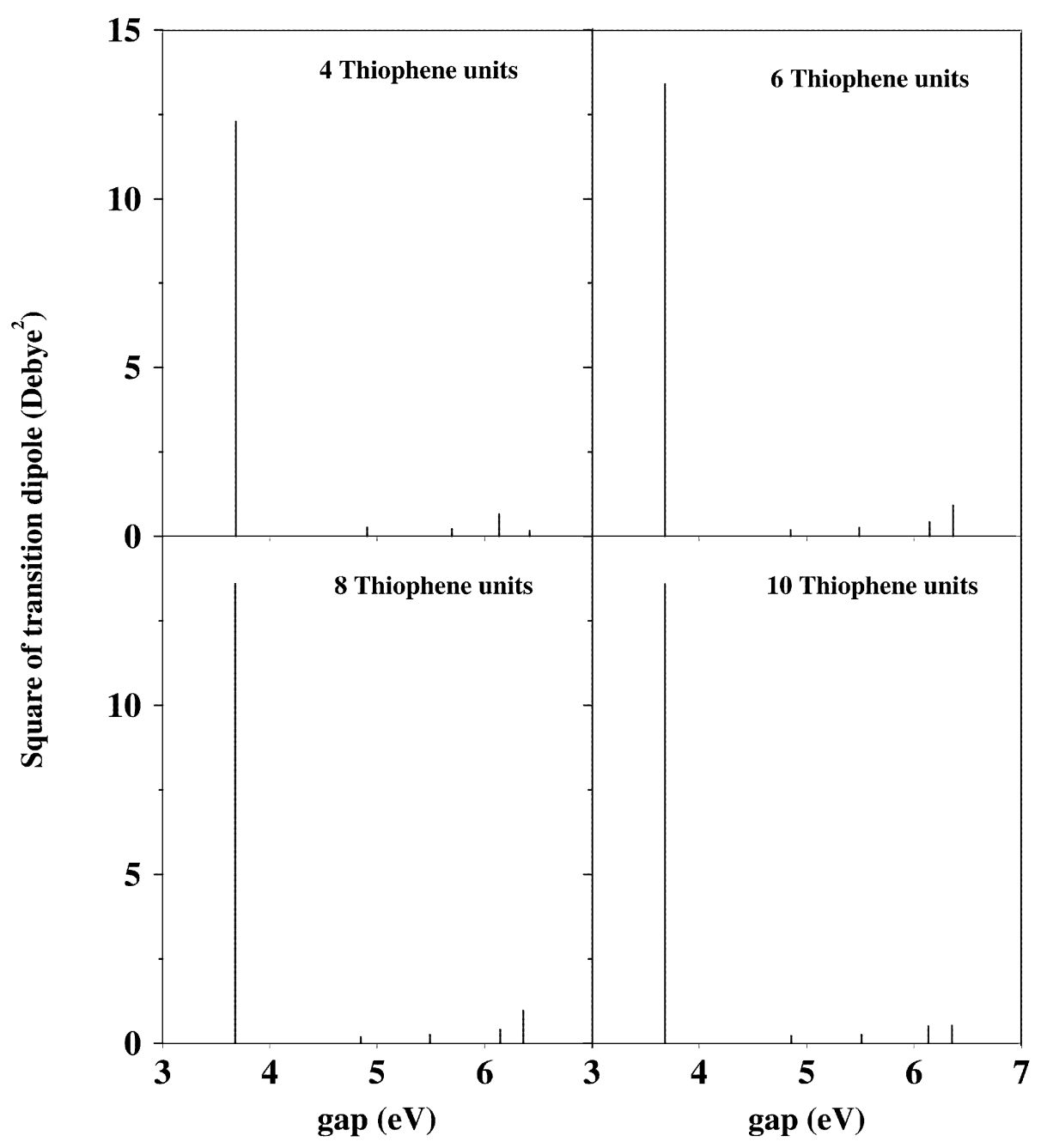

Figure 10. Square of the transition dipole plot as a function of energy gaps for thiophene oligomers with up to 10 monomers.

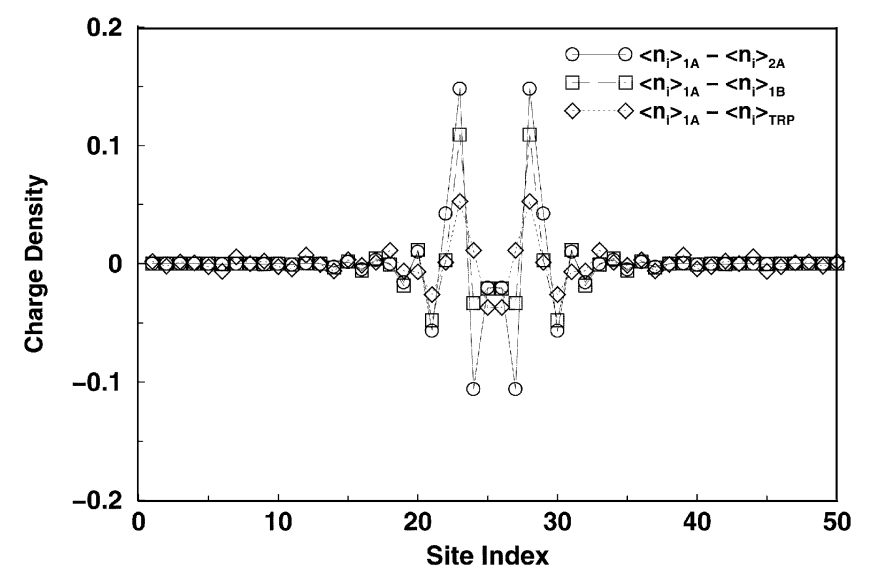

Figure 11. Comparison of differences in charge density of one-photon, two-photon and lowest triplet states. The difference in charge density is taken with respect to that of the ground state. ment of the charge density takes place only around the center of the molecule, the charge density variation with respect to ground state is nearly zero at both ends of the polymer. According to the site numbering illustrated in figure 4 , we can clearly see that in case of one-photon excitation, charge transfer takes place from sulphur to carbon atoms. This process can be represented by the resonance structures shown in figure 12 , with the weight of the ionic structure being larger in the one-photon state. The resonance structure implies increase in the $\mathrm{C}-\mathrm{S}$ bond order and decrease in the neighbouring $\mathrm{C}-\mathrm{C}$ bond-order, relative to the ground state. This is indeed seen in the bond-order plots (figure 13). The two-photon state has a larger weight of the ionic structure as evidenced by higher charges on the carbons and sulphur atoms. The bond-order pattern of the two-photon state 
is similar to the one-photon state. Thus unlike in polyenes where the two-photon state is delocalized and the one-photon state is localized, in PT both the excitations appear to be localized excitations.

The bond orders and charge densities in the triplet state also indicate that the ionic resonance structures have significant weight in the triplet state. The magnitude of charge transfer from the sulphur atom to carbon is however least for the triplet state, of all the states discussed. The triplet excitation is also slightly more delocalized than the other two excitations. The resonance structures also imply that large spin density should reside on the sites where negative charge resides for the one- and two-photon states. In all the

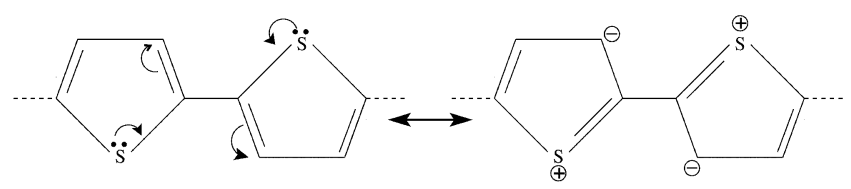

Figure 12. Resonance structure indicates the charge transfer taking place from sulphur to carbon atom at the middle of the polymer.
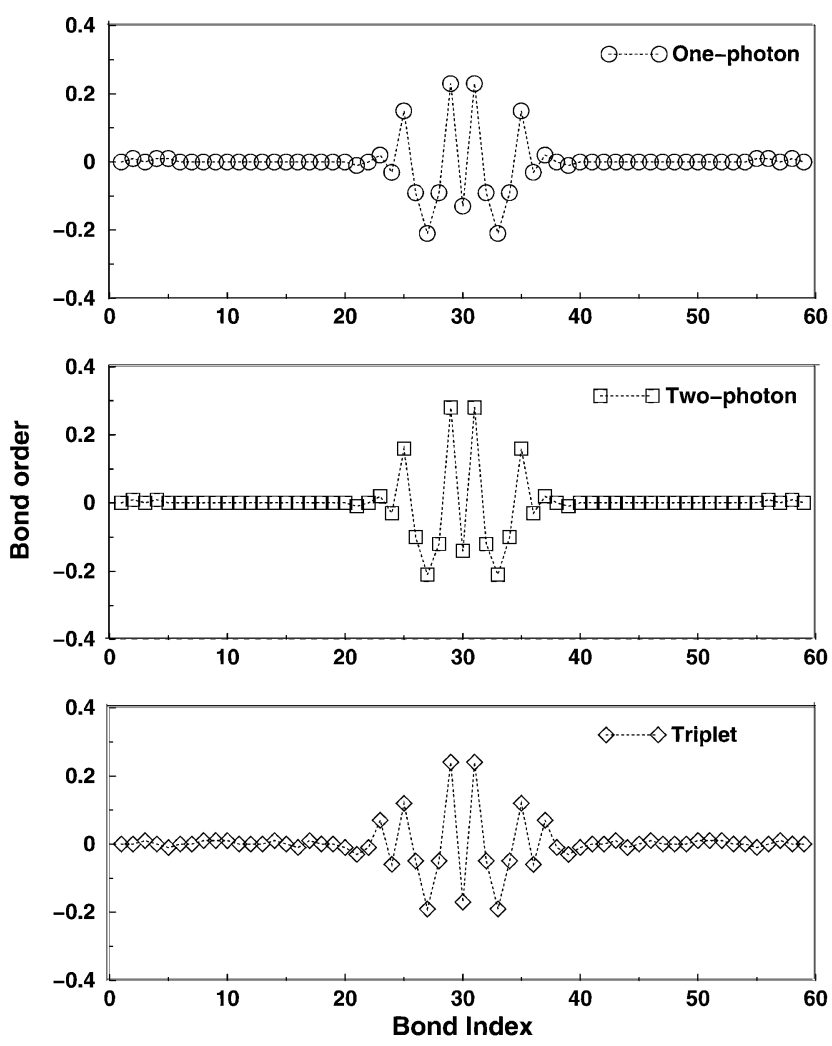

Figure 13. The difference in bond orders of one-photon state $\left(1^{1} B\right)$, two-photon state $\left(2^{1} A\right)$, and lowest triplet state $\left(1^{3} B\right)$ with respect to the ground state $\left(1^{1} A\right)$ for 10 thiophene rings. cases studied above, we can get some insights into the equilibrium geometry of the excited states from the bond order data. Bond distances in the equilibrium geometry are shorter than the bond distances in the ground state, if the corresponding bond order in the given excited state is larger than that in the ground state. We note in all cases that the change in bond orders from the ground state is localized in the middle of the oligomer. We also note that the distributions within triplet excitation is more spread out than in other excited states.

\section{Doped polymer}

The primary process for electrical conduction is to create a pair of freely moving electron and hole. Such a process can be achieved by exciting an electron across the band gap by using light and thus can be monitored by the measurement of photoconductivity as a function of wavelength of incident light. The photoconductivity threshold, known as the charge gap, can be computed by representing the physical process as,

$\mathrm{M} \stackrel{h v}{\longrightarrow} P^{+}+P^{-}$

where $\mathrm{M}$ is the polymer in the ground state and $P^{+} / P^{-}$are the positively and negatively charged polarons created by the incident light. The energy of the above process can be computed by extrapolating to the thermodynamic limit, the charge gap, $E_{c}(N)$ defined as,

$$
E_{c}(N)=E_{P-}(N)+E_{P^{+}}(N)-2 E_{\mathrm{M}}(N),
$$

where $E_{\mathrm{M}}(N), E_{P-}(N), E_{P^{+}}(N)$ are the energies in the ground state of neutral, negatively charged and positively charged $N$-site oligomers respectively. In this we have assumed that in the thermodynamic limit $(N \rightarrow \infty) E_{\mathrm{M}}(2 N)=2 E_{\mathrm{M}}(N)$. We have calculated the charge gap for oligomers with up to 10 thiophene rings. From figure 14 the extrapolated value of the charge gap is $7.80 \mathrm{eV}$ which is very large. The charge gap gives the photoconductivity threshold of the polymer. The optical excitation, on the other hand, need not give rise to well separated charges as in the photoconductivity process, since the optically excited state could correspond to the bound $P^{+} P^{-}$state, called the excitonic state. The upper bound for the excitonic binding energy is given by the difference between the charge gap $E_{c}$ and the optical gap $E_{g}$ 
(see figure 14). The charge gap and the photo-excitation gap coincide in the Hubbard model provided that all the transfer integrals are uniform. If the electron-electron interactions are extended beyond onsite, the electron and the hole could form a bound state by residing in the vicinity of one another, leading to an exciton. Exciton formation takes place only in $U-V$ or PPP models. In such situation, the optical gap is not same as the charge gap. In the band picture, the excitonic levels are found below the conduction band edge as shown in figure 15 . From the value of extrapolated optical gap which is $3.67 \mathrm{eV}$, we find the binding energy of the $1^{1} B^{-}$exciton as $4.13 \mathrm{eV}$, which is very large, compared to polyenes or polypara phenylene vinylenes (PPV).

Overdoping (underdoping) the conjugated polymers can lead to addition (removal) of two electrons rather than one. If both the electrons (holes) stay to-

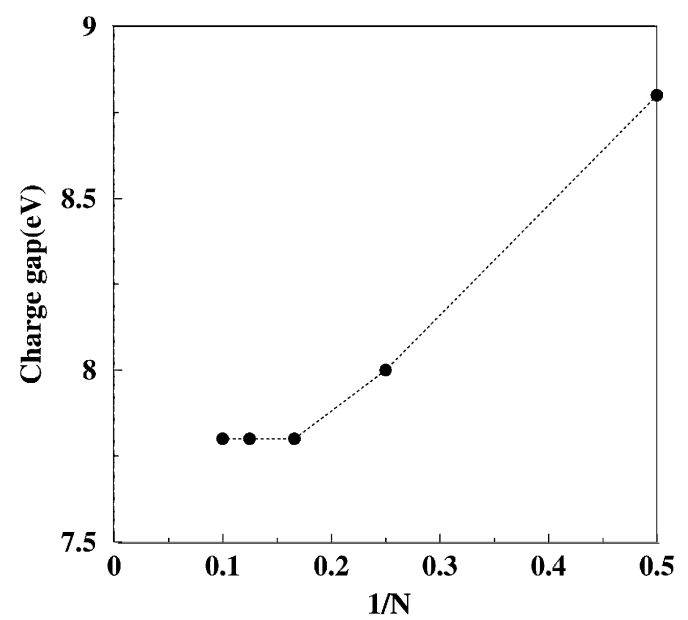

Figure 14. The charge gap for polythiophene as a function of inverse of system size.

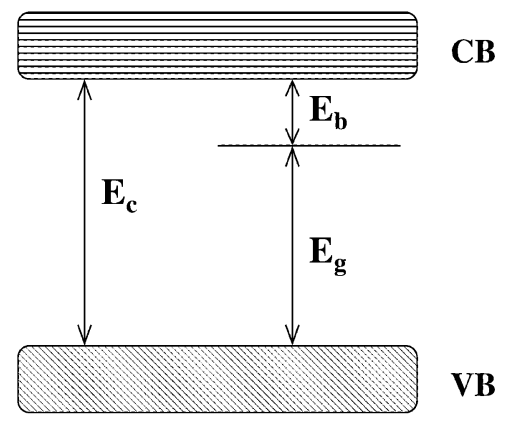

Figure 15. Schematic representation of a single excitonic level below conduction band, $E_{c}, E_{g}, E_{b}$ are the charge gap, the optical gap and the exciton binding energy. VB and $\mathrm{CB}$ represent the valence and conduction bands respectively. gether then we have the formation of a bipolaron. In order to investigate whether the bipolaron is a stable entity or it breaks up into two polarons, we have calculated the binding energy of the bipolaron, along the lines described earlier for the excitons. The binding energy can be defined as $\varepsilon_{b p}=E_{N}\left(P^{++}\right)+E_{N}(\mathrm{M})-$ $2 E_{N}\left(P^{+}\right)$of the reaction process given by,

$$
P^{+}+P^{+} \rightarrow P^{++}+\mathrm{M}
$$

If the binding energy of the bipolaron is negative in the thermodynamic limit, the bipolaron is stable. We have calculated the stabilization energy as defined above by infinite DMRG algorithm and have plotted binding energy vs $1 / N$ in figure 16 . We obtain $\varepsilon_{b p}=+0.85 \mathrm{eV}$, in the thermodynamic limit showing

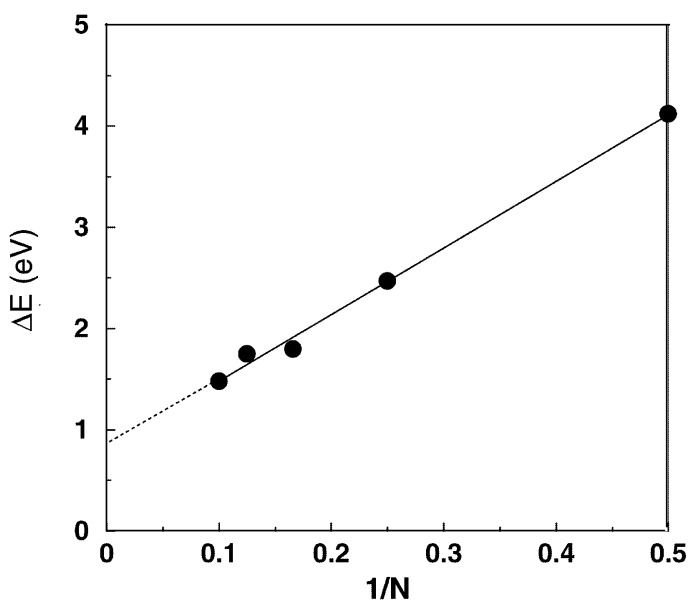

Figure 16. The binding energy of the bipolaron, as a function of inverse system size.

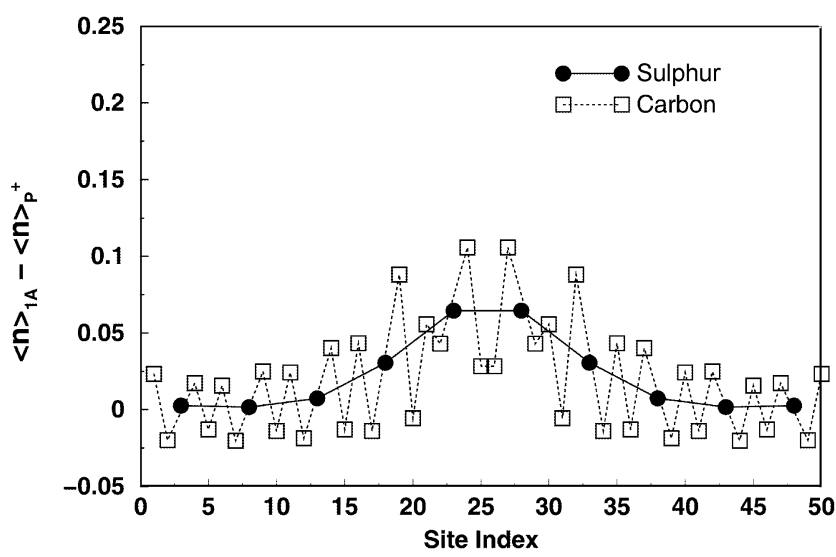

Figure 17. The difference in the charge density for polaron with respect to that of the ground state for 10 thiophene rings. 


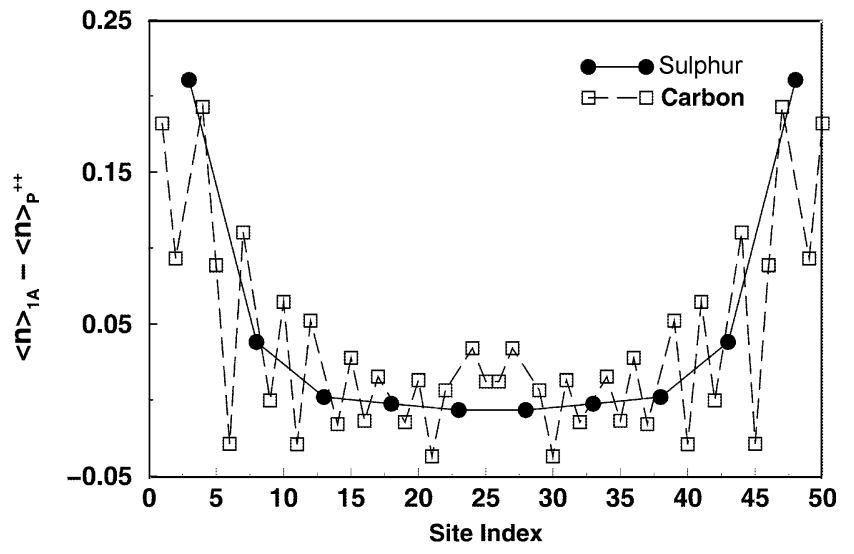

Figure 18. The difference in the charge density for bipolaron with respect to that of the ground state for 10 thiophene rings.

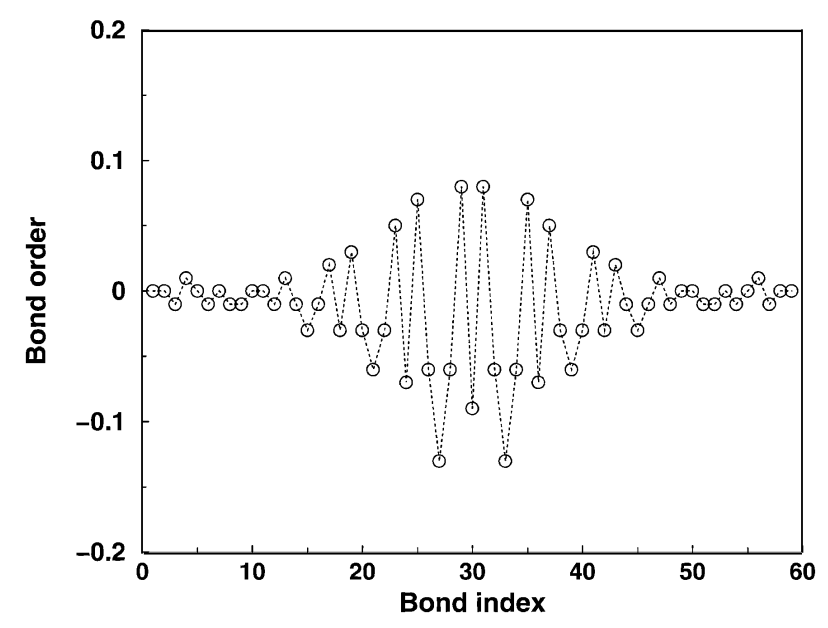

Figure 19. The difference in bond orders of polaronic state with respect to the ground state $\left(1^{1} A\right)$ for 10 thiophene rings.

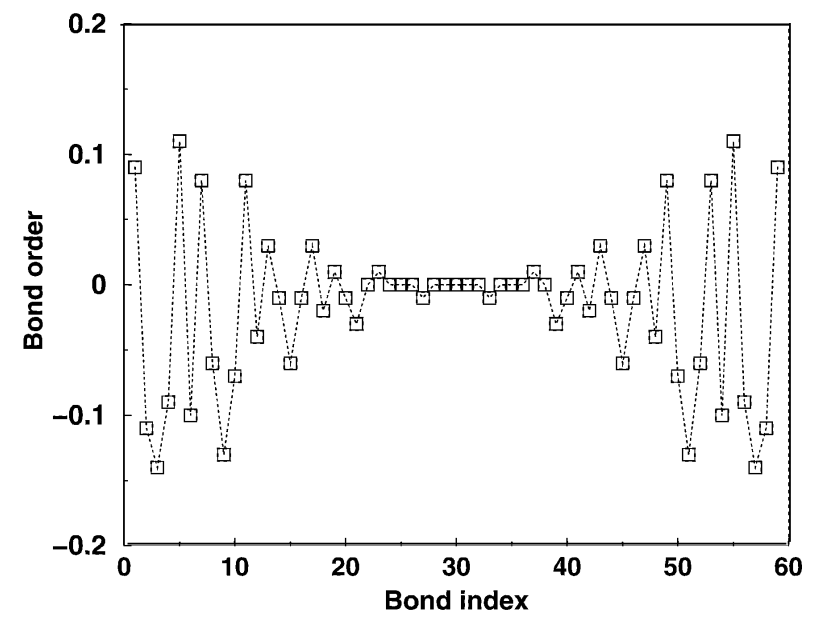

Figure 20. The difference in bond orders of bipolaronic state with respect to the ground state $\left(1^{1} A\right)$ for 10 thiophene rings. that the bipolaron is not formed in polythiophene. For further verification of the absence of the biplaronic state, we have calculated the charge density for both polaron and bipolaron. The calculations are for system size of 10 thiophene rings. We have plotted the differences in charge density of polaron and bipolaron with respect to the charge density of the ground state in figures 17 and 18 respectively. The difference in charge density has been shown separately for carbon and sulphur atoms. Figure 17 shows that a significant difference in the charge density in case of polarons occurs mostly in the middle of the system for both carbon and sulphur. There is redistribution of charges at carbon atoms. Within a ring, the alternate carbon atoms have positive and negative charges compared to the neutral ground state, whereas charges are depleted from the sulphur atoms. From the figure we observe that the polaron is delocalized over 4 thiophene rings. This result is in agreement with the earlier work by Brédas et $a l,{ }^{51}$ although their study showed that the participation of sulphur atom is negligible in polaron formation. From our study, we see that sulphur atoms indeed participate in the polaron formation, but to a lesser extent than the carbon atoms. In contrast to polaron, bipolaron is more localized. We observe that bipolaron splits into two polarons and the charges are separated and piled up at the ends of the chain (figure 18). This can be attributed to the Coulombic repulsion between two positively charged polarons. This agrees with our prediction from the calculation of binding energy that bipolarons are not a stable entity.

Figures 19 and 20 show the bond order difference of the doped polaronic state and bipolaronic state with respect to ground state. There is maximum fluctuation in bond order in the middle of the chain for polaronic state and at the ends of the chain for bipolaronic state. Polaron bond order is delocalized over 4 rings of polythiophene. This reinforces the fact that the doubly doped chain leads to two separated polarons instead of a single bipolaron. The nature of bond order pattern for polarons is similar to that of one-photon and two-photon excited states, the polaronic state however is more delocalized.

\section{Conclusions}

We have studied the nature of the ground state and the low-lying excited states of thiophene oligomers with up to 10 thiophene rings using symmetrized density matrix renormalization group technique within 
the long-range PPP model Hamiltonian. We find that the lowest dipole-allowed state lies below the lowest forbidden two-photon state. This result implies polythiophene is strongly fluorescent in agreement with experiments. The lowest triplet state lies below the two-photon state which also agrees with the general trend in conjugated polymers. Besides computing optical gap and two-photon gap, we have computed the charge gap of the system. We have estimated the binding energy of $1^{1} B^{-}$exciton to be $4.13 \mathrm{eV}$. We have calculated the charge densities of the ground state, one-photon state and two-photon state and computed the bond orders of the lowest excited states along with the ground state. These studies indicate that these three excited states are localized and have similar charge redistribution with respect to the ground state. We have also studied doped systems with singly and doubly doped oligomers. The charge density and bond order for singly doped and doubly doped states for system size of 10 thiophene rings show that the bipolarons are not stable and dissociate into two polarons. This is in agreement with the binding energy calculation for bipolaron.

\section{Acknowledgement}

The authors are grateful to the Department of Science and Technology, Government of India, for financial support.

\section{References}

1. Taliani C and Gebauer W 1999 Handbook of oligo and polthiophenes (ed.) Fichou D (Weineheim: Wiley-VCH) and references therein

2. Roncall J 1992 J. Chem. Rev. 92711

3. Patil A O, Heeger A J and Wudl F 1988 Chem. Rev. 88183

4. Horowitz G, Deloffre F, Garnier F, Hajlaoul R, Hmyene M and Yassar A 1993 Synth. Met. 54435

5. Ostoja P, Goerri S, Rossini S, Servidor M, Taliani C and Zaboni R 1993 Synth. Met. 54447

6. Burroughes J H, Bradeley D D C, Brown A R, Marks R N, Machay K, Friend R H, Burns P L and Holmes A B 1990 Nature (London) 347539

7. Berggren M, Inganäs O, Rasmusson J, Gustafsson G, Andersson M R, Wennerström $\mathrm{O}$ and Hjertberg $\mathrm{T}$ 1994 Nature (London) 372444

8. Tessler N, Denton G J and Friend R H 1996 Nature (London) $\mathbf{3 8 2} 695$

9. Granlund T, Theander M, Berggren M, Andersson M R, Ruseckas A, Sundström V, Björk G, Granström M and Inganäs O 1998 Chem. Phys. Lett. 288879
10. Halls J J M, Pichler K, Friend R H, Moratti S C and Holmes A B 1996 Appl. Phys. Lett. 683120

11. Yu G and Heeger A J 1995 J. Appl. Phys. 784510

12. Garnier F, Hajilaoui R, Yassar A and Srivastava P 1994 Science 2651684

13. Dodabalapur A, Torsi L and Katz H E 1995 Science 268270

14. Dodabalapur A, Katz H E, Torsi L and Haddon R C 1995 Science 2691560

15. Periasamy N, Danieli R, Ruani G, Zamboni R and Taliani C 1992 Phys. Rev. Lett. 68919

16. Pfeffer N, Raimond P, Charra F and Nunci J M 1993 Chem. Phys. Lett. 201357

17. Swanson L S, Lane P A, Shinar J and Wudl F 1991 Phys. Rev. B44 10617

18. Soos Z G, Etemad S, Galvao D S and Ramasesha S 1992 Chem. Phys. Lett. 194341

19. Birnbaum D and Kohler E 1989 J. Chem. Phys. 90 3506

20. Birnbaum D and Kohler B E 1991 J. Chem. Phys. 95 4783

21. Birnbaum D, Fichou D and Kohler B E $1992 J$. Chem. Phys. 96165

22. Birnbaum D and Kohler B E 1992 J. Chem. Phys. 96 2492

23. Wheland G W and Pauling L 1935 J. Am. Chem. Soc. $\mathbf{5 7} 2086$

24. Schomaker V and Pauling L 1939 J. Am. Chem. Soc. 611769

25. Beljonne D, Shuai Z and Brédas J L 1993 J. Chem. Phys. 98, 8819

26. Negri F and Zgierski M Z 1994 J. Chem. Phys. 100 2571

27. Soos Z G and Galvo D S 1994 J. Phys. Chem. 98 1029

28. Colditz R, Grebner D, Helbig M and Rentsch S 1995 Chem. Phys. 201309

29. Becker R S, Sexias de Melo J, Macanita A L and Elisei F 1996 J. Phys. Chem. 10018683

30. Solony N, Birss F W and Greenshields J B 1965 Can. J. Chem. 43, 1569

31. Bielefeld H J and Fits D D 1966 J. Am. Chem. Soc. 884804

32. Billingsley F P and Bloor J E 1968 Theor. Chim. Acta 11325

33. Tajiri A, Asano T and Nakajima T 1968 Tetrahedron Lett. 211785

34. Mehlhorn M, Schwenzer B and Schwtlick K 1977 Tetrahedron 331483

35. Bendazzoli G L, Bertinelli F, Palmieri P, Brillante A and Taliani C 1978 J. Chem. Phys. 695077

36. Matsushita M, Tanaka H and Nishimoto K 1983 Theor. Chim. Acta 6355

37. Serrano-Andrés L, Merchán M, Fülscher M and Roos B O 1983 Chem. Phys. Lett. 211125

38. Pariser R and Parr R G 1953 J. Chem. Phys. 21 446; Pople J A 1953 Trans. Faraday Soc. 491375

39. Mazumdar S 1992 Conjugated conducting polymers (ed.) H G Kiess (Berlin: Springer-Verlag)

40. White S R 1992 Phys. Rev. Lett. 692863 
41. Peschel I, Wang X, Kaulke M and Hallberg K 1999 Density-matrix renormalization: A new numerical method in physics (Lecture notes in physics) (Berlin: Springer)

42. Ramasesha S, Pati S K, Krishnamurthy H R, Shuai Z and Brédas J L 1996 Phys. Rev. B54 7598

43. Ohno K 1964 Theor. Chem. Acta 2219

44. Callomon J H, Hirota E, Kuchitsu K, Lafferty W J, Maki A G and Pote C S 1976 Landolt-Bornstein, numerical data and functional relationships in science and technology, New Series, Group II: Atomic and molecular physics, structure data of free polyatomic molecules (Berlin-Heidelberg: Springer-Verlag) vol 7
45. Patil A O and Heeger A J and Wudl F 1988 Chem. Rev. 88183

46. Ducasse L R, Miller T E and Soos Z G 1982 J. Chem. Phys. 764094

47. Soos Z G and Ramasesha S 2002 Valence bond theory (ed.) D L Cooper (Amsterdam: Elsevier Science), ch. 20, p 635

48. Kasha M 1950 Discuss. Faraday Soc. 914

49. Thienpont H, Rikken G L J A, Meijer E W, ten Hoeve W and Wynberg H 1990 Phys. Rev. Lett. 652141

50. Monkman A P, Burrows H D, Hartwell L J, Horsburgh L E, Hamblett I and Navaratnam S 2001 Phys. Rev. Lett. 861358

51. Stafström S and Brédas J L 1988 Phys. Rev. B38 4180 